\title{
A CONGRUENCE FOR THE SIGNATURE OF AN EMBEDDED MANIFOLD
}

\author{
ROBERT D. LITTLE
}

(Communicated by Frederick R. Cohen)

\begin{abstract}
Let $M^{2 n}$ be a smooth, closed, orientable $2 n$-manifold and suppose that $K_{x}^{2 n-2}$ is an orientable submanifold of $M^{2 n}$ dual to a cohomology class $x$. If $d$ is a positive integer, the signatures of $K_{d x}^{2 n-2}$ and $K_{x}^{2 n-2}$ are related by a numerical congruence. If $n$ is odd, then any codimension 2 submanifold of $\mathbf{C} P^{n}$ fixed by a diffeomorphism of odd prime order is dual to the generator of the cohomology algebra.
\end{abstract}

\section{INTRODUCTION}

Let $M^{2 n}$ be a smooth, closed, orientable $2 n$-manifold and suppose that $x \in H^{2}(M ; \mathbf{Z})$. Let $K_{x}^{2 n-2}$ be an orientable codimension 2 submanifold of $M$ dual to $x$; that is, if $i: K_{x} \subset M$ is the inclusion map, then $i_{*}\left[K_{x}\right]=x \cap[M]$. A theorem of Thom asserts that such a submanifold can be found for any cohomology class $x$, [12, Théorèm II.27]. If $n$ is odd and $d$ is a nonnegative integer, then the signatures of $K_{d x}$ and $K_{x}$ are related by a numerical congruence. If $m$ is an integer, let $\nu_{2}(m)$ be the exponent of 2 in the prime decomposition of $m$.

Theorem 1.1. If $n$ is odd and $d$ is a nonnegative integer, then for any $x$ in $H^{2}(M ; \mathbf{Z})$,

$$
n ! \operatorname{Sign} K_{d x} \equiv n ! d \operatorname{Sign} K_{x}\left(\bmod 2^{\nu_{2}(n !)} d\left(1-d^{2}\right)\right) .
$$

It is natural to apply formula (1.1) to $\mathbf{C} P^{n}$, complex projective $n$-space, with $x \in H^{2}\left(\mathbf{C} P^{n} ; \mathbf{Z}\right)$ the generator of the cohomology algebra. Every orientable codimension 2 submanifold of $\mathbf{C} P^{n}$ is dual to $d x$ for some integer $d$, the degree of the submanifold. We will always assume that the submanifold is oriented so that $d \geq 0$. Let $p$ be an odd prime and $G_{p}$ denote the cyclic group of order $p$. Suppose that $F$ is a codimension 2 submanifold of $\mathbf{C} P^{n}$

Received by the editors August 30, 1989; presented August 17, 1990 at Topology Hawaii, a conference sponsored by the National Science Foundation and the University of Hawaii.

1980 Mathematics Subject Classification (1985 Revision). Primary 55Q50, 55R50, 55S25, 57Q25, 57R10.

Key words and phrases. Signature, homotopy complex projective space, group actions. 
fixed by a $G_{p}$ action on $\mathbf{C} P^{n}$. The fixed point set of the action is equal to $F$ and an isolated point, [3, Corollary 0.1$]$. It has been conjectured that the degree of $F$ is $1,[8$, p. 589]. This follows from (1.1) for $n$ odd.

Theorem 1.2. If $n$ is odd and $F$ is a codimension 2 submanifold of $\mathbf{C} P^{n}$ fixed by a smooth $G_{p}$ action, then the degree of $F$ is 1 .

Theorem 1.1 can be used to obtain information about the degree of a fixed submanifold in other cases. If $M^{2 n}$ is a $\mathbf{Z}$-cohomology $\mathbf{C} P^{n}$, let $x \in H^{2}(M ; \mathbf{Z})$ be the generator of the cohomology algebra.

Theorem 1.3. Suppose that $n$ is odd and that $M^{2 n}$ is a Z-cohomology $\mathbf{C} P^{n}$. If $M^{2 n}$ supports a smooth $G_{p}$ action fixing a codimension 2 submanifold of degree $d$, then $d$ is an odd divisor of $n !$ and $d=1$ if and only if there is a submanifold dual to $x$ with signature 1 . If $M^{2 n}$ is a homotopy $\mathbf{C} P^{n}$, then $d \equiv 1(\bmod 8)$ and $d$ divides $n$ !.

Note that Theorem 1.2 follows immediately from Theorem 1.3 , for if $n$ is odd, then $\mathbf{C} P^{n-1}$ is a submanifold of $\mathbf{C} P^{n}$ which is dual to $x$ and has signature 1. Another immediate consequence of Theorem 1.3 is

Theorem 1.4. If $n=3$ or 5 and $M^{2 n}$ is a smooth homotopy $\mathbf{C} P^{n}$, then the degree of a codimension 2 submanifold of $M^{2 n}$ fixed by a smooth $G_{p}$ action is 1 .

The above theorems seem to support the conjecture that if $n$ is odd and $M^{2 n}$ is a smooth homotopy $\mathbf{C} P^{n}$, then the only codimension 2 submanifolds of $M^{2 n}$ fixed by a $G_{p}$ action are those of degree 1 . If this conjecture is true, we will see that (1.1) implies the existence of a smooth homotopy $\mathbf{C} P^{n}$ which does not support a smooth $G_{p}$ action fixing a codimension 2 submanifold for any odd prime $p$. If $n \equiv 1(\bmod 4)$, then at least this consequence of the truth of the conjecture is true.

Theorem 1.5. If $n \equiv 1(\bmod 4)$, then there exists a smooth homotopy $\mathbf{C} P^{n}$ which does not support a smooth $G_{p}$ action fixing a codimension 2 submanifold for any odd prime $p$.

Petrie conjectured that a smooth homotopy $\mathbf{C} P^{n}, M^{2 n}$, which supports an effective circle action must have a standard Pontrjagin class; that is, $p_{*}\left(M^{2 n}\right)=$ $\left(1+x^{2}\right)^{n+1},[10]$. This conjecture is true in the special case where the circle action fixes a codimension 2 submanifold. In fact, if a $\mathbf{Q}$-cohomology $\mathbf{C} P^{n}$ supports a circle action fixing a codimension 2 submanifold, then it must have a standard Pontrjagin class and the degree of the fixed submanifold is $1,[8$, Theorem A]. Less is known about codimension 2 fixed submanifolds of $G_{p}$ actions. If $n=3$ or 4 and $M^{2 n}$ is a smooth homotopy $\mathbf{C} P^{n}$ which supports a $G_{p}$ action of this type, then the Pontrjagin class of $M^{2 n}$ is standard and the degree of the fixed submanifold is $1,[3$, Theorem A; 6, p. 4]. Theorem 1.2 and 
Theorem 1.4 in the case $n=5$ give other examples where the degree of the fixed submanifold is 1 .

We offer a final application of (1.1) to an entirely different situation. If $n$ is even and $M^{2 n}$ supports a smooth $G_{p}$ action such that the fixed point set is equal to a codimension 2 submanifold (possibly not connected), then $p$ divides Sign $M^{2 n}$ if $p>n+1$, [4, Theorem 4.5]. Formula (1.1) can be used to study this kind of action on submanifolds of $\mathbf{C} P^{n+1}$.

Theorem 1.6. Suppose that $n$ is even and $M^{2 n}$ is a submanifold of $\mathbf{C} P^{n+1}$ of degree $d$. If $p>n+1$ and $d^{2} \equiv 1(\bmod p)$, then $M^{2 n}$ does not support a smooth $G_{p}$ action with fixed point set a codimension 2 submanifold.

For example, if $d \geq 2$ let $V_{d}^{2 n}=\left\{\left[z_{0}, z_{1}, \ldots, z_{n+1}\right] \in \mathbf{C} P^{n+1}: z_{0}^{d}+z_{1}^{d}+\cdots+\right.$ $\left.z_{n+1}^{d}=0\right\}$. If $n$ is even, $p>n+1$, and $d^{2} \equiv 1(\bmod p)$, then $V_{d}^{2 n}$ does not support a smooth $G_{p}$ action with fixed point set a codimension 2 submanifold. Note that $V_{d}^{2 n}$ does support a $G_{d}$ action of this type.

The paper is organized as follows. In $\S 2$, we prove Theorem 1.1. In $\S 3$, we study smooth $G_{p}$ actions on a Z-cohomology $\mathbf{C} P^{n}$ which fix a codimension 2 submanifold and we prove the first statement in Theorem 1.3. Section 4 is devoted to smooth homotopy complex projective $n$-spaces and it contains the proof of the second statement in Theorem 1.3 as well as the proof of Theorem 1.5. In $\S 5$, we discuss $G_{p}$ actions and prove Theorem 1.6.

\section{The SignATURE CONGRUENCE}

The idea of expressing Sign $K_{d x}$ as a polynomial function of $d$ and $x$ is not new [13]. These polynomials tend to be difficult to compute if $n$ is large and one way around this difficulty is to produce an integral congruence valid for any $n$. In our first proposition, we have the hypotheses of Theorem 1.1 that $n$ is odd and that $K_{x}$ is dual to $x \in H^{2}\left(M^{2 n} ; \mathbf{Z}\right)$. If $d$ is a nonnegative integer and $z$ is a complex number, let

$$
f_{d}(z)=\left[(1+z)^{d}-(1-z)^{d}\right] /\left[(1+z)^{d}+(1-z)^{d}\right] .
$$

Proposition 2.1. If $f_{d}(z)=\sum_{k=0}^{\infty} r_{k} z^{2 k+1}$, then

$$
\operatorname{Sign} K_{d x}=r_{0} \operatorname{Sign} K_{x}+\sum_{k=1}^{(n-1) / 2} r_{k} \operatorname{Sign} K_{x}^{(2 k+1)},
$$

where $K_{x}^{(2 k+1)}$ is the $2 k+1$-fold selfintersection of $K_{x}$ in $M^{2 n}$.

Proof. The argument begins by noting that

$$
\text { Sign } K_{d x}=\{\tanh d x L(M)\}[M],
$$

where $L(M)$ is the Hirzebruch $L$-class of $M,[13,(1.1)]$. The identity $\tanh d x$ $=f_{d}(\tanh x),[4$, p. 208] implies that

$$
\operatorname{Sign} K_{d x}=\left\{\sum_{k=0}^{\infty} r_{k}(\tanh x)^{2 k+1} L(M)\right\}[M] .
$$


Formula (2.2) follows from (2.4) because

$$
\left\{(\tanh x)^{2 k+1} L(M)\right\}[M]=\operatorname{Sign} K_{x}^{(2 k+1)},
$$

([13], (8.3) in the special case $N=M$ and $d=1)$. The summation terminates at $k=(n-1) / 2$ because the dimension of $K_{x}^{(2 k+1)}$ is $2 n-4 k-2$.

Proposition 2.1 makes it clear that Theorem 1.1 will follow from an analysis of the coefficients $r_{k}$ in the power series of $f(z)$. If $m$ is a positive integer, let $\operatorname{odd}(m)=2^{-\nu_{2}(m)} m$.

Proposition 2.2. The coefficients $r_{k}, 0 \leq k<+\infty$, are polynomial functions of $d$ with rational coefficients such that $r_{0}(d)=d$ and if $k \geq 1$, then $r_{k}(d)=$ $d\left(1-d^{2}\right) q_{k}\left(d^{2}\right)$, where $q_{k}\left(d^{2}\right)$ is a rational polynomial in $d^{2}$ such that $\operatorname{odd}((2 k+1) !) q_{k}\left(d^{2}\right)$ is a polynomial in $d^{2}$ with integer coefficients.

Before proceeding with the proof of Proposition 2.2, we make some preliminary observations. It follows from the binomial expansion formula and (2.1) that $r_{0}(d)=d$ and if $k \geq 1$, then $r_{k}(d)$ is a rational polynomial in $d$ such that

$$
r_{k}(d)=\left(\begin{array}{c}
d \\
2 k+1
\end{array}\right)-r_{k-1}(d)\left(\begin{array}{l}
d \\
2
\end{array}\right)-r_{k-2}(d)\left(\begin{array}{l}
d \\
4
\end{array}\right)-\cdots-d\left(\begin{array}{c}
d \\
2 k
\end{array}\right) .
$$

The proof of Proposition 2.2 is organized in three lemmas. The first lemma shows that the proposition is valid for $k=1,2,3$ and gives explicit values for $q_{k}\left(d^{2}\right)$. The second lemma uses an inductive argument based on the first lemma to show that $(2 k+1) ! r_{k}(d)$ is always an integral polynomial in $d$. The third lemma shows that the denominators of the coefficients of $r_{k}(d)$ are odd by showing that there is an odd number $\mu_{k}$ such that $\mu_{k} r_{k}(d)=d\left(1-d^{2}\right) s_{k}\left(d^{2}\right)$, where $s_{k}\left(d^{2}\right)$ is a polynomial in $d^{2}$ with integer coefficients.

Lemma 2.3. If $k=1,2$, or 3 , then $r_{k}(d)=d\left(1-d^{2}\right) q_{k}\left(d^{2}\right)$, where

$$
q_{k}\left(d^{2}\right)= \begin{cases}1 / 3, & k=1, \\ \left(3-2 d^{2}\right) / 15, & k=2, \\ \left(17 d^{4}-53 d^{2}+45\right) / 315, & k=3 .\end{cases}
$$

Proof. This is a straightforward computation based on (2.5). Note that $\operatorname{odd}((2 k+1) !) q_{k}\left(d^{2}\right)$ is an integral polynomial in $d^{2}$ if $k=1,2$, or 3 .

Lemma 2.4. If $k \geq 1$, then $(2 k+1) ! r_{k}(d)$ is a polynomial in $d$ with integer coefficients.

Proof. The proof is by induction. The statement of the lemma is true for $k=1,2,3$ by Lemma 2.3 , and so let $k>3$ and assume inductively that $(2 j+1) ! r_{j}(d)$ is a polynomial in $d$ with integer coefficients for $1 \leq j \leq k-1$. It follows that $(2 j+1) !(2 k-2 j) ! r_{j}(d)\left(\begin{array}{c}d \\ 2 k-2 j\end{array}\right)$ is an integral polynomial in $d$ for $1 \leq j \leq k-1$. The inductive step in the proof, that is, the fact that 
$(2 k+1) ! r_{k}(d)$ is an integral polynomial in $d$, follows from this observation, (2.5), and the fact that $(2 j+1) !(2 k-2 j)$ ! divides $(2 k+1)$ ! .

The next step is to show that only odd factors of $(2 k+1)$ ! are needed in Lemma 2.4 and to produce the factorization of $r_{k}(d)$ mentioned in Proposition 2.2. It would be interesting to have an analytical proof of this fact based on (2.5), but at present, we only have an argument based on Lemma 2.4 and an application of (2.2) to a universal example. If $k$ is a positive integer, let

$$
\mu_{k}=\prod_{\text {primes } p>2} p^{[2 k /(p-1)]} \text {. }
$$

For example, $\mu_{1}=3, \mu_{2}=45$, and $\mu_{3}=945$. The number $\mu_{k}$ has the property that if $L_{k}\left(P_{1}, P_{2}, \ldots, P_{k}\right)$ is the Hirzebruch $L$-polynomial, then $L_{k}^{\prime}\left(P_{1}, P_{2}, \ldots, P_{k}\right)=\mu_{k} L_{k}\left(P_{1}, P_{2}, \ldots, P_{k}\right)$ is a polynomial with coprime integer coefficients, [2, p. 39; 9 , p. 225]. Note that if $p$ is a prime, the exponent of $p$ in $m$ ! satisfies the equation $\nu_{p}(m !)=\left(m-\lambda_{p}(m)\right) /(p-1)$, where $\lambda_{p}(m)$ is the sum of the digits in the $p$-adic decomposition of $m$, and so $\operatorname{odd}((2 k+1) !)$ divides $\mu_{k}$.

Lemma 2.5. If $k \geq 1$, then $\mu_{k} r_{k}(d)=d\left(1-d^{2}\right) s_{k}\left(d^{2}\right)$, where $s_{k}\left(d^{2}\right)$ is a polynomial in $d^{2}$ with integer coefficients.

Proof. The proof is by induction. Lemma 2.3 guarantees that the statement in Lemma 2.5 is true for $k=1,2,3$ and so if $k>3$, assume inductively that $\mu_{j} r_{j}(d)=d\left(1-d^{2}\right) s_{j}\left(d^{2}\right), 1 \leq j \leq k-1$, where $s_{j}\left(d^{2}\right)$ is an integral polynomial in $d^{2}$. We will prove the inductive step by applying formula (2.2) to the embedding $K_{d x}^{4 k} \subset \mathbf{C} P^{2 k+1}$ to obtain

$$
\operatorname{Sign} K_{d x}^{4 k}=r_{0}(d)+\sum_{j=1}^{k} r_{j}(d) .
$$

Formula (2.8) has this simple form because the selfintersections in formula (2.2) satisfy the equations $K_{x}^{(2 j+1)}=\mathbf{C} P^{2 k-2 j}, 1 \leq j \leq k$, in this particular case.

Lemma 2.5 at level $k$ will follow from (2.8) and the inductive hypothesis if we can show that there is a polynomial in $d^{2}$ with the integer coefficients, $t_{k}\left(d^{2}\right)$, such that

$$
\mu_{k} \operatorname{Sign} K_{d x}^{4 k}=\mu_{k} d+d\left(1-d^{2}\right) t_{k}\left(d^{2}\right) .
$$

Formula (2.9) is just (4.2) in [7] in the special case of a codimension 2 submanifold of $\mathbf{C} P^{2 k+1}$. With (2.9) in hand the inductive step in the proof follows from (2.8) and the inductive hypothesis since $r_{0}(d)=d$.

Proof of Proposition 2.2. The only thing left to prove is that if $k \geq 1$, then $r_{k}(d)=d\left(1-d^{2}\right) q_{k}\left(d^{2}\right)$, where $\operatorname{odd}((2 k+1) !) q_{k}\left(d^{2}\right)$ is a polynomial in $d^{2}$ with integer coefficients. It follows from Lemmas 2.4 and 2.5 that if $k \geq 1$, 
then $\operatorname{odd}((2 k+1) !) r_{k}(d)$ is an integral polynomial in $d$, and because $\mu_{k}$ is divisible by odd $((2 k+1) !)$, we have

$$
\left(\mu_{k} / \operatorname{odd}((2 k+1) !)\right) \operatorname{odd}((2 k+1) !) r_{k}(d)=d\left(1-d^{2}\right) s_{k}\left(d^{2}\right) .
$$

So the coefficients of $s_{k}\left(d^{2}\right)$ are all divisible by the integer $\mu_{k} / \operatorname{odd}((2 k+1) !)$, and if $\hat{s}_{k}\left(d^{2}\right)$ denotes $s_{k}\left(d^{2}\right)$ divided by this integer, then the factorization of the proposition holds with $q_{k}\left(d^{2}\right)=\hat{s}_{k}\left(d^{2}\right) / \operatorname{odd}((2 k+1) !)$.

Proof of Theorem 1.1. Formula (1.2) follows immediately from (2.2) after multiplying both sides of (2.2) by $n$ ! and invoking Proposition 2.2.

We close this section with applications of (1.1). The first application is to submanifolds of $\mathbf{C} P^{n}, n$ odd. Formula (1.1) gives a congruence for the signature of a submanifold of degree $d$ because $K_{x}=\mathrm{C} P^{n-1}$ and so $\operatorname{Sign} K_{x}=1$.

Theorem 2.6. If $n$ is odd and $K^{2 n-2} \subset \mathbf{C} P^{n}$ is a submanifold of degree $d$, then

$$
n ! \operatorname{Sign} K \equiv n ! d \quad\left(\bmod 2^{\nu_{2}(n !)} d\left(1-d^{2}\right)\right) .
$$

The second application is to submanifolds of $V_{d}^{2 n}, n \geq 3$. If $i: V_{d}^{2 n} \subset$ $\mathbf{C} P^{n+1}$ is the inclusion map, then $i^{*}: H^{k}\left(\mathbf{C} P^{n+1} ; \mathbf{Z}\right) \rightarrow H^{k}\left(V_{d}^{2 n} ; \mathbf{Z}\right)$ is an isomorphism for every $k \neq n$ and the rank of $H^{n}\left(V_{d}^{2 n} ; \mathbf{Z}\right)$, that is, the Betti number $\beta_{n}$, depends on $n$ and $d,[13, \mathrm{p} .71]$. In particular, there is a notion of degree for codimension 2 submanifolds of $V_{d}^{2 n}$ because $H^{2}\left(V_{d}^{2 n} ; \mathbf{Z}\right) \cong \mathbf{Z}$. The submanifold of $V_{d}^{2 n}$ dual to the generator of $H^{2}\left(V_{d}^{2 n} ; \mathbf{Z}\right)$ is $V_{d}^{2 n-2}$. If $n$ is odd, (1.1) can be applied to $V_{d}^{2 n}$ with $K_{x}=V_{d}^{2 n-2}$ and a congruence for $n$ ! Sign $V_{d}^{2 n-2}$ given by $(2.10)$.

Theorem 2.7. If $n$ is odd and $K^{2 n-2} \subset V_{d}^{2 n}$ is a submanifold of degree $D$, then

$$
n ! \operatorname{Sign} K \equiv n ! D d \quad\left(\bmod 2^{\nu_{2}(n !)} D \operatorname{gcd}\left(d\left(1-d^{2}\right),\left(1-D^{2}\right)\right)\right) .
$$

\section{Actions of $G_{p}$ FIXING A CODIMENSION 2 SUBMANIFOLd}

In this section, $M^{2 n}$ is a $\mathbf{Z}$-cohomology $\mathbf{C} P^{n}$, that is, a smooth, closed orientable $2 n$-manifold with $H^{*}(M ; \mathbf{Z})=\mathbf{Z}[x] /\left(x^{n+1}\right)$ where $x \in H^{2}(M ; \mathbf{Z})$. If $G_{p}$ acts smoothly on $M^{2 n}$ in such a way that the fixed point set $F\left(M^{2 n}\right)$ contains a codimension 2 submanifold $F$, then $F\left(M^{2 n}\right)=F \cup$ point , [3, Corollary 0.1 . In this paper, we will restrict ourselves to a discussion of the codimension 2 component of the fixed point set. The behavior of the action at the isolated point is discussed in $[3,7]$. The $\mathbf{Z}_{p}$-cohomological structure of $F$ is well understood: $H^{*}\left(F ; \mathbf{Z}_{p}\right) \cong H^{*}\left(\mathbf{C} P^{n-1} ; \mathbf{Z}_{p}\right),[1, \mathbf{p} .378]$. Over the ring of integers, everything is known modulo torsion. If $H$ is an abelian group, let $H_{f}=H /$ torsion and if $x \in H$, let $f(x)$ be the image of $x$ in $H_{f}$. If 
$i: F \subset M^{2 n}$ and $\hat{x}=i^{*}(x)$, then $H^{*}(F ; \mathbf{Z})$ contains the truncated polynomial ring $\mathbf{Z}[f(\hat{x})] /\left(f\left(\hat{x}^{n}\right)\right)$, [3, Corollary 0.1$]$, and the degree of $F$ is given by $d=f\left(\hat{x}^{n-1}\right)[F],[3,(\mathrm{a})$, p. 503]. The next lemma is Lemma 5.2 in [7].

Lemma 3.1. If $n$ is odd, then $\operatorname{Sign} F= \pm 1$ and the orientation of $F$ may be so chosen that $\operatorname{Sign} F= \pm 1$ and the degree of $F$ is positive.

Theorem 3.2. Suppose that $n$ is odd and that $M^{2 n}$ supports a smooth $G_{p}$ action fixing a codimension 2 submanifold $F$. If the degree of $F$ is $d$, then

$$
\operatorname{odd}(n !) \equiv \operatorname{odd}(n !) d \operatorname{Sign} K_{x} \quad\left(\bmod d\left(1-d^{2}\right)\right) .
$$

In particular, $d$ is an odd divisor of $n !$ and $d=1$ if and only if $M^{2 n}$ contains a submanifold dual to $x$ with signature 1 .

Proof. Formula (3.1) follows immediately from (1.1) and Lemma 3.1. It is also clear that formula (3.1) implies that $d$ is an odd divisor of $n$ !. If $d=1$, it is clear from (3.1) that $\operatorname{Sign} K_{x}=1$. Conversely, suppose that $K_{x}$ is a submanifold dual to $x$ such that $\operatorname{Sign} K_{x}=1$. Formula (3.1) implies that there is an integer $m$ such that $\operatorname{odd}(n !)(1-d)=m d\left(1-d^{2}\right)$, where $d>0$. If we assume that $d \neq 1$, we obtain $\operatorname{odd}(n !)=m d(d+1)$ by dividing, which is impossible.

Theorem 3.2 constitutes a proof of the first statement in Theorem 1.3. If every $\mathbf{Z}$-cohomology $\mathbf{C} P^{n}$ contained a submanifold dual to $x$ with signature 1 , (3.1) would imply $d=1$. We will see that not every homotopy $\mathbf{C} P^{n}$ contains this type of submanifold dual to $x$.

\section{SMOOTH HOMOTOPY COMPLEX PROJECTIVE SPACES}

In this section, $M^{2 n}$ is a smooth homotopy $\mathbf{C} P^{n}$, that is, a smooth, closed $2 n$-manifold together with a hornotopy equivalence $h: M^{2 n} \rightarrow \mathbf{C} P^{n}$. If $h$ is put into general position with respect to $\mathbf{C} P^{n-1} \subset \mathbf{C} P^{n}$ and we let $K=$ $h^{-1}\left(\mathbf{C} P^{n-1}\right)$, it is clear from naturality that $K$ is dual to $x$, that is, $K=K_{x}$. There are surgery problems $h \mid K_{x}^{(n-i)}: K_{x}^{(n-i)} \rightarrow \mathbf{C} P^{i}$ and associated surgery obstructions $\sigma_{i}$, where $i$ is an integer such that $0 \leq i \leq n-1$. If $i$ is even, then $\sigma_{i}$ is an integer such that $\operatorname{Sign} K_{x}^{(n-i)}=1+8 \sigma_{i}$, and if $i$ is odd, then $\sigma_{i}$ is a mod 2 integer, [11, p. 6]. Note that $\sigma_{i}=s_{2 i}$, where $s_{2 i}$ is a Sullivan splitting invariant. These splitting invariants determine the PL homeomorphism type of $M^{2 n},[14,14 C .2$.$] . The splitting invariant \sigma_{0}$ is zero because $x^{n}$ is the cofundamental class and so $\operatorname{Sign} K_{x}^{(n)}=1$. If $n$ is odd, the surgery problems $h \mid K_{x}^{(2 k+1)}: K_{x}^{(2 k+1)} \rightarrow \mathbf{C} P^{n-2 k-1}, 0 \leq k \leq(n-1) / 2$, determine integral splitting invariants $\sigma_{n-2 k-1}$ such that $\operatorname{Sign} K_{x}^{(2 k+1)}=1+8 \sigma_{n-2 k-1}$. The next two theorems are immediate consequences of the above remarks, (1.1), (2.2), and Theorem 3.2. They apply the signature formulas to the case of a homotopy $\mathbf{C} P^{n}$. 
Theorem 4.1. If $n$ is odd and $K^{2 n-2} \subset M^{2 n}$ is a submanifold of degree $d$, then

$$
\begin{gathered}
\operatorname{Sign} K=\sum_{k=0}^{(n-1) / 2} r_{k}(d)\left(1+8 \sigma_{n-2 k-1}\right), \\
\operatorname{odd}(n !) \operatorname{Sign} K \equiv \operatorname{odd}(n !) d\left(1+8 \sigma_{n-1}\right) \quad\left(\bmod d\left(1-d^{2}\right)\right) .
\end{gathered}
$$

Theorem 4.2. If $n$ is odd and $M^{2 n}$ supports a smooth $G_{p}$ action fixing a codimension 2 submanifold of degree $d$, then

$$
\operatorname{odd}(n !) \equiv \operatorname{odd}(n !) d\left(1+8 \sigma_{n-1}\right) \quad\left(\bmod d\left(1-d^{2}\right)\right) .
$$

In particular, $d \equiv 1(\bmod 8), d$ divides $n !$, and $d=1$ if and only if $\sigma_{n-1}=0$.

Proof of Theorem 1.3. The proof of the first statement in Theorem 1.3 is contained in Theorem 3.2. The statement about homotopy projective spaces is contained in Theorem 4.2 .

If $n$ is odd and $M^{2 n}$ is a smooth homotopy $\mathbf{C} P^{n}$ with $\sigma_{n-1}=0$ (e.g., any PL suspension, $[14,14 C .3]$, such as the standard $\mathbf{C} P^{n}$ ), then the degree of any codimension 2 submanifold fixed by a $G_{p}$ action is 1 . Theorem 4.2 shows that if the conjecture is true that $d$ is 1 , then any $M^{2 n}$ with $n$ odd and $\sigma_{n-1} \neq 0$ does not support a smooth $G_{p}$ action fixing a codimension 2 submanifold for any odd prime $p$. We are not able to prove the conjecture for $n>5$, but we can give examples of smooth homotopy projective spaces which do not admit $G_{p}$ actions of this type. If $n$ is a positive integer, let $E(n)=\max \left\{\nu_{2}(m-1): m\right.$ divides $\operatorname{odd}(n !)\}$.

Lemma 4.3. If $n$ is odd and $M^{2 n}$ is a smooth homotopy $\mathbf{C} P^{n}$ such that $\sigma_{n-1}$ is a nonzero multiple of $2^{E(n)-2}$, then $M^{2 n}$ does not support a smooth $G_{p}$ action fixing a codimension 2 submanifold for any odd prime $p$.

Proof. Suppose that the action exists and let $d$ be the degree of the fixed submanifold. Since $\sigma_{n-1} \neq 0$, we know that $d>1$ by Theorem 4.2. We will show that no such value of $d$ can satisfy (4.3). First, we assume that (4.3) holds and we let $e=\nu_{2}(d-1)$. Theorem 4.2 implies that $3 \leq e \leq E(n)$. Formula (4.3) implies that there is an integer $s$ such that $\operatorname{odd}(n !)(1-d)=$ $8 d \operatorname{odd}(n !) \sigma_{n-1}+s d\left(1-d^{2}\right)$. This is a contradiction since our choice of $\sigma_{n-1}$ implies that the left side of the equation is divisible by $2^{e}$ but not divisible by $2^{e+1}$ while the right side of the equation is divisible by $2^{e+1}$.

The next step is to construct the smooth homotopy projective space in Lemma 4.3. The hypothesis of smoothability restricts the integral splitting invariants. If $i$ is a positive integer, let $B_{i}$ denote the $i$ th Bernoulli number, [9, p. 281], and let $m_{i}=2^{2 i-2}\left(2^{2 i-1}-1\right) \operatorname{num}\left(B_{i} / 4 i\right)$, where $\operatorname{num}\left(B_{i} / 4 i\right)$ is the numerator of the rational number $B_{i} / 4 i$ reduced to lowest terms. For example, $m_{1}=1$, 
$m_{2}=28$, and $m_{3}=496,\left[9\right.$, p. 285]. If $M^{2 n}$ is a smooth homotopy $\mathbf{C} P^{n}$ and $n=4,5$, or 6 , then the splitting invariants of $M^{2 n}$ must satisfy certain numerical congruences modulo $m_{2}$ and $m_{3}$, [6, Theorems 1.1, 1.2, 1.3]. In high dimensions, there is a construction theorem, [7, Theorem 6.1]: if $i$ is even and $m \equiv 0\left(\bmod 2 m_{i}\right)$, then there exists a smooth homotopy $\mathbf{C} P^{2 i+1}$ such that $\sigma_{2 j}=0,1 \leq j \leq i-1$, and $\sigma_{2 i}=m$.

Proof of Theorem 1.5. If $n \equiv 1(\bmod 4)$, let $n=2 i+1$ with $i$ even. Let $t$ be a positive integer with $\nu_{2}(t) \geq E(2 i+1)-(2 i+1)$ and let $M^{4 i+2}$ be a smooth homotopy $\mathbf{C} P^{2 i+1}$ such that $\sigma_{2 i}=2 t m_{i}$. It follows from Lemma 4.3 that $M^{4 i+2}$ does not support a smooth $G_{p}$ action fixing a codimension 2 submanifold for any odd prime $p$.

\section{HATTORI'S THEOREM}

In this section, $M^{2 n}$ denotes a smooth, closed orientable $2 n$-manifold with $n$ even. If $m$ is an integer such that each odd prime factor of $m$ is greater than $n+1$ and $M^{2 n}$ admits a smooth, semi-free action of $G_{m}$ by orientation preserving diffeomorphisms such that the fixed point set has codimension 2 and is orientable, then the oriented bordism class of $M^{2 n}$ is divisible by $m,[4$, Theorem 4.5]. In particular, $m$ divides $\operatorname{Sign} M^{2 n}$. To say that $M^{2 n}$ supports a $G_{m}$ action of the type in Hattori's theorem is to say that $M^{2 n}$ is an $m$-fold cyclic ramified branched covering with orientable branching locus, [4, p. 208]. If $p$ is an odd prime, $G_{p}$ actions necessarily preserve orientation and have orientable fixed point sets, and so Theorem 1.6 is a consequence of the stronger

Theorem 5.1. Suppose that $n$ is even and that $M^{2 n}$ is a submanifold of $\mathbf{C} P^{n+1}$ of degree $d$. If each odd prime factor of $m$ is greater than $n+1$ and $d^{2} \equiv 1$ $(\bmod m)$, then $M^{2 n}$ is not an $m$-fold cyclic ramified branched covering with orientable branching locus.

Proof. It follows from $(2.10)$ that $\operatorname{odd}((n+1) !) \operatorname{Sign} M^{2 n} \equiv \operatorname{odd}((n+1) !) d$ $\left(\bmod d\left(1-d^{2}\right)\right)$. If $d^{2} \equiv 1(\bmod m)$ and $M^{2 n}$ is an $m$-fold cyclic ramified branched covering space with orientable branching locus, Hattori's theorem leads to the contradiction $\operatorname{odd}((n+1) !) d \equiv 0(\bmod m)$.

A weaker version of (1.1) appeared in [7]. This version of the formula was established only for Z-cohomology complex projective space and the coefficient $\operatorname{odd}(n !)$ was replaced by $\mu_{(n-1) / 2}[7,(4.2)]$. The locally linear PL versions of Theorems 1.2 and 1.4 are discussed in [7] and Theorem 1.4 is extended to the case $n=7$ in the smooth category.

\section{ACKNOWLEDGMENT}

The author is greatly indebted to Robert E. Stong for suggesting that the preliminary version of (1.1) could be replaced by a general signature congruence. In particular, Proposition 2.1 is due to Stong. The author would like to 
thank the referee for mentioning certain unpublished work of $F$. Connolly and S. Weinberger. Connolly and Weinberger studied codimension 2 submanifolds of $\mathbf{C} P^{n}$ which have the property that they are fixed by an action of a cyclic group. They may actually have had a result which implies Theorem 1.2.

\section{REFERENCES}

1. G. E. Bredon, Introduction to compact transformation groups, Academic Press, London, 1972.

2. G. Brumfiel, On integral PL characteristic classes, Topology 8 (1969), 39-46.

3. K. H. Dovermann, Rigid cyclic group actions on cohomology projective spaces, Math. Proc. Cambridge Philos. Soc. 101 (1987), 487-507.

4. A. Hattori, Genera of ramified coverings, Math. Ann. 195 (1972), 208-226.

5. D. Husemoller, Fibre bundles, Springer-Verlag, New York, 1975.

6. R. D. Little, Homotopy complex projective spaces with divisible splitting invariants, Pacific J. Math. 140 (1989), 251-262.

7. _ The defect of codimension two submanifolds of homotopy complex projective space, $\mathrm{J}$. London Math. Soc. (to appear).

8. M. Masuda, Smooth group actions on cohomology complex projective spaces with a fixed point component of codimension 2, A fête of topology, Academic Press, Boston, 1988, pp. 585-602.

9. J. W. Milnor and J. D. Stasheff, Characteristic classes, Ann. of Math. Studies, No. 76, Princeton University Press, Princeton, 1974.

10. T. Petrie, Smooth $S^{1}$ actions on homotopy complex projective spaces and related topics, Bull. Amer. Math. Soc. 78 (1972), 105-153.

11. D. Sullivan, Triangulating and smoothing homotopy equivalences and homeomorphisms, Geometric topology seminar notes, Princeton University, 1967.

12. R. Thom, Quelques propriétés globales des variétés differentiables, Comm. Math. Helv. 28 (1954), 17-86.

13. E. Thomas and J. Wood, On manifolds representing homology classes in codimension 2, Invent. Math. 25 (1974), 63-89.

14. C.T.C. Wall, Surgery on compact manifolds, Academic Press, London, 1970.

Department of Mathematics, University of Hawail at Manoa, Honolulu, Hawail 96822 